\title{
A New User-Apps Interactions Dataset for Behavioral Profiling Using Smartphones
}

\author{
Saud Alotaibi ${ }^{1}$, Abdulrahman Alruban ${ }^{1,2}$, Moneerah Alotaibi ${ }^{1,3}$, Ali Alshumrani ${ }^{1,4}$ \\ Abdulaziz Altamimi ${ }^{1}$ \\ ${ }^{1}$ Centre for Security, Communications and Network Research, Plymouth University, Plymouth, UK \\ ${ }^{2}$ Computer Sciences and Information Technology College, Majmaah University, Al Majma'ah, Saudi Arabia \\ ${ }^{3}$ Computer Science Department, Shaqra University, Shaqra, Saudi Arabia \\ ${ }^{4}$ College of Computers and Information Systems, Umm Al-Qura University
}

\begin{abstract}
The use of mobile devices in our daily lives has grown steadily. These mobile devices contain sensitive data such as text messages, photos, communication logs, contact lists, personal information and stored passwords. They are also used to perform activities such as sending emails or transferring money via mobile Internet banking, which is considered a sensitive process. As a consequence, more security is needed on mobile devices since, after point-of-entry authentication, the user can perform almost all tasks without having to re-authenticate. On the other hand, many authentication methods have been suggested to improve the security of mobile devices in a transparent and continuous manner, providing a basis for convenient and secure user reauthentication. In addition, although a number of studies have investigated the feasibility of using behavioural biometrics to secure a mobile device, there is a lack of studying user behavioural profiling interactions with their smartphones due to there are no such datasets available. The main aim of this paper is to present a new user-apps Interactions dataset for behavioral profiling using Smartphones which might help researchers to improve smartphones security. A study involving data collected from 76 users over a 1-month period was conducted, generating over 3 million actions based on users' interactions with their smartphone. This study also demonstrates and highlights some future work by utilizing the acquired dataset to provide robust, continuous and transparent authentication and usable system as well.
\end{abstract}

\section{Introduction}

The use of mobile devices in our daily lives has grown steadily, due to the combination of mobility and 24/7 multi-connectivity. In particular, smartphones are used to perform activities, such as sending emails, transferring money via mobile Internet banking, making calls, texting, surfing the Internet, viewing documents, storing medical, confidential and personal information, shopping online and playing games. As a result, mobile devices have overtaken desktop computers. For instance, the total number of smartphone users worldwide from 2014 to projections for 2022 [1]. In addition, the number of smartphone users in 2019 is forecast to pass 5 billion. For example, in the USA, the number is forecast to grow to 247.5 million by 2019 [1]. Globally, there are 3.419 billion people connected to the Internet (equating to $46 \%$ global penetration), while 2.307 billion users are actively involved in social media. In addition, 3.790 billion people are unique mobile users (representing 51\% global penetration), whereas 1.968 billion users utilise social media on a mobile device [2].

Statista [2] predicted that, in 2020, the expected growth of mobile app revenue would be $\$ 101$ billion, from $\$ 41.1$ billion in 2015 . It was expected that revenue from mobile apps would grow at a steady rate in the coming years. Moreover, mobile web traffic was expected to exceed 10 exabyte by 2017 [3]. Regarding the digital marketing review [4], mobile devices achieve $75 \%$ of all adults' time online with smartphones. On the other hand, $80 \%$ of female spend their time on mobile devices compare with only $69 \%$ for males and $30 \%$ of online adults are now mobile only as well. Based on audience, over $90 \%$ of time online is spent on smartphones for Spotify and Snapchat whereas tablets account for over a third of time spent on the BBC as illustrated in Figure 2 2. In addition, YouTube increased both its mobile app audience (about 5\%) and time spent (about 22\%) compared to 2017. Interestingly, Snap is the only mobile app in the top 10 which is not owned by Google or Facebook. Furthermore, Spotify, Netflix and eBay feature in the top 10 mobile apps for time spent [4].

Currently, sensitive data such as text messages, contact lists, and personal information are stored on mobile devices. This makes authentication of paramount importance. More security is needed on mobile devices since, after point-of-entry authentication, the user can perform almost all tasks without having to re-authenticate. [5, 6]. As a result, there is an urgent need to verify the identity of the current user of a mobile device. It must be possible to authenticate legitimate users and detect imposters in a continuous and transparent manner, maintained 
beyond point-of-entry, without the explicit involvement of the user [7]. To this end, data on user behaviour is gathered in the background without requiring any dedicated activity by the user, by regularly and periodically checking user behaviour in order to continuously monitor the protection of the mobile device [8]. In addition, although a number of studies have investigated the feasibility of using behavioural biometrics to secure a mobile device, there is a lack of user action interactions with their smartphones dataset to investigate the behavioural profiling, as shown in Table 1 . This research study considered this and collected a total of 3,015,339 actions which might be helpful for the researchers.

The next section presents related work and the state of the art of smartphone behaviour profiling biometrics. This is followed by an outline of a data collection methodology to smartphone security in section 3. Then, section 4 presents data collection analysis with more explanations and discussions. Finally, section 5 concludes the paper.

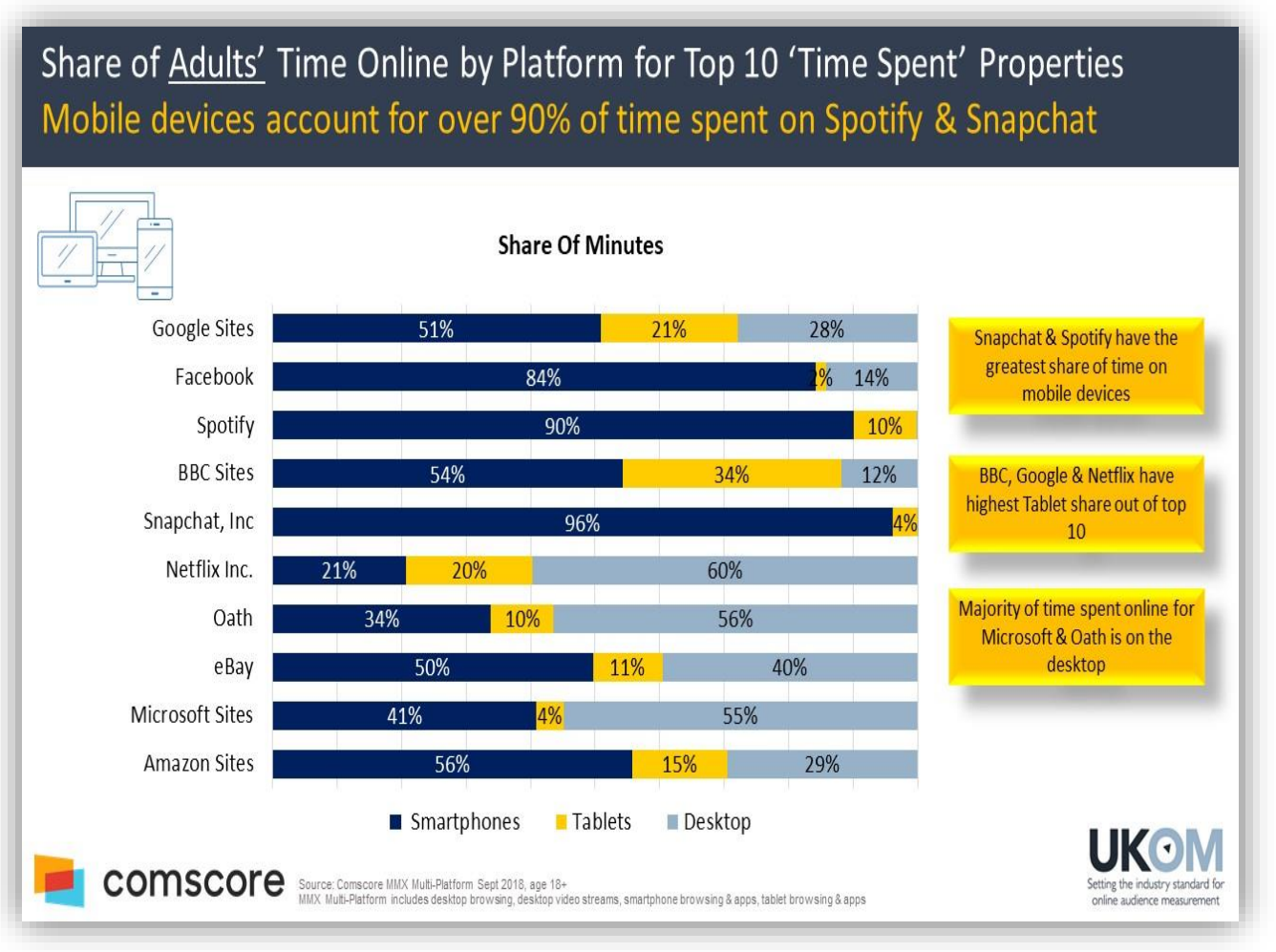

Figure 1. UK digital marketing review [4]

\section{Related Work}

It is commonly acknowledged that biometric authentication is a reliable solution to authenticating users using convenient and trusted methods [9, 10]. Most biometric authentication systems are capable of providing a wide range of transparent authentication approaches to achieve a high level of balance between usability and security [6]. In this context, behavioural biometrics is often presented as a suitable authentication method and, indeed, is commonly used for transparent and continuous authentication while ensuring usability $[5,11]$. One type of behavioural biometric is behaviour profiling. The main aim in this case is the transparent verification of mobile users based on the way they interact with the required service whilst using their smartphone [5,12]. This approach compares the current user's activities with a historical profile of usage that is built utilising a machine learning method [13].

Although a limited number of studies have focused on behavioural profiling-based authentication for mobile devices, some investigative efforts have been made in the literature to introduce behavioural profiling as a behavioural biometrics authentication approach to providing transparent authentication [6]. For instance, $\mathrm{Li}$ et al. [14] introduced a behaviour profiling approach to identify mobile device misuse by focusing on the mobile user's application usage. This work used the MIT Reality Mining dataset [15]. The following data were collected from 100 smartphone users for 9 months: application information (app name, date, duration of usage and cell ID), voice call data (including date, time, number called, duration, and cell ID), and text message data (date, time, number texted and cell 
ID) $[12,13]$. Later, the authors presented a novel behaviour profiling framework that was able to collect user behaviour to evaluate the system security status of a device in a continuous manner before sensitive services were accessed [16]. They investigated the sensitivity of the application concept, which is mapped to high-risk levels to make the framework more secure and transparent when the user requires access to high-risk applications. The authors concluded that the approach seems able to distinguish mobile users through their application usage; in particular, by focusing on the names of applications and the location of usage, which are considered valuable features.

Among further studies in a similar context, Saevanee et al. [17] examined the combination of three diverse biometric methods: keystroke dynamics, behavioural profiling and linguistic profiling from 30 virtual users (the dataset was not real and was gathered from different datasets). To continue their work, Saevanee et al. [18] presented a text-based authentication framework utilising the above modalities and introduced a security aspect by allowing the user to set security levels for access to different applications. In other recent work, Fridman et al. [19] proposed a parallel binary decision-level fusion architecture for active authentication. The fusion is used for classifiers based on four biometric modalities: text analysis, application usage patterns, web browsing behaviour, and the physical location of the device through GPS (outdoors) or Wi-Fi (indoors). To evaluate the framework, the authors collected a dataset from 200 users' Android mobile devices over a period of 5 months.

In the same context, Neal and Woodard [20] introduced associative classification to authenticate mobile device users by analyzing the performance of applications. Bluetooth and Wi-Fi data were collected from 189 college-level students over 19 months. Three time intervals $(5,15$, and $30 \mathrm{~min})$ were selected and association rules were extracted from each data type separately and combined as features. Prior to that, Shi et al. [21] recorded users' routines, such as location, phone calls, and application usage, in order to build a profile and assign a positive (e.g., good behaviour, such as a phone call to a known number) or negative score for each user's routine, using a dataset based on 50 users for a period of 12 days or more. The dataset contained SMS, phone call, browser history and location, without demonstrating the finding of this study.

To conclude, there is a lack of user action interactions with their smartphones dataset to investigate the behavioural profiling, as shown in Table 1.

Table 1. Datasets results

\begin{tabular}{|l|l|l|l|}
\hline Study & \# of Subject & Dataset & Features \\
\hline Shi et al. , (2011) [21] & 50 for & 12 days & SMS, Calls, Browser History,GPS \\
\hline Hayashi et al., (2012) [23] & 20 & Structured interview & - \\
\hline Gupta et al., (2012) [24] & $37-76$ & MIT Reality & GPS location, WIFI, Bluetooth \\
\hline Li et al., (2014) [16] & $22-76$ & MIT Reality & App name, Tel. number, cell, GPS \\
\hline Khan and Heng., (2014)[22] & 30 & 4different datasets & - \\
\hline Kayacik et al, (2014) [25] & $7,35,100$ & GCU, MIT Reality & Wi-Fi, CPU load, light, noise \\
\hline Fridman et al. (2015) [19] & 200 & 30 days & Text, app usage, Wi-Fi, GPS \\
\hline Neal and Woodard (2017) [20] & 189 & students & Bluetooth and Wi-Fi data \\
\hline Acien et al .(2019) [26] & 48 & Maryland University & App usage, GPS, Wi-Fi, Touch \\
\hline
\end{tabular}

Note: $\mathrm{n} / \mathrm{a}=$ not applicable

On the other hand, predicting mobile app usage has motivated researchers in the past, there are some research in this area. Tan et al. [28] conducted experiments on the Nokia MDC dataset that involves 38 users and proposed an algorithm to predict mobile application usage patterns. Huang et al. [29] discuss the faster execution of desired app through the pre-loading of the right apps in memory or through app pop up to the mobile's home screen. They exploit a set of feature such as time, location, and the user profile, to predict the user's app usage using the Nokia MDC dataset already mentioned. Yan et al. [30] developed app preloading method that use contextual information such as user location and temporal access patterns to predict app launches with 34 users. Also, Zou et al. [31] developed some light-weighted Bayesian models that use the app usage history to predict the next app that user is going to use. Liao et al. [32] developed a widget that use temporal profiles which identify the relation between apps and their usage times in order to predict user's app usage. In addition, Pan et al. [33] used social information coming from friends of the user in a social networks to predict the most likely mobile application that a user will install. Krishnaswamy 
et al. [34] developed a different prediction method that discovers frequent co-occurrence patterns on the phone to indicate to the context events that frequently occur together.

\section{Mobile Data Collection Methodology}

In order to investigate the feasibility of building a transparent and continuous biometric-based system, it is necessary to collect samples of genuine user interactions with their mobile devices/apps, based upon a substantive period of real-world use (noting that such samples would be based upon data that are naturally logged by apps on the devices already and so the research would not be gathering information that was not already available - it would, however, be applying it to an additional purpose). As such, it was proposed to enlist participants and collect log data from them after one month of normal device usage. It should be noted that the data were anonymous and that participation did not require the participants to do anything other than use their devices as normal. This experiment collected the sort of data that are logged routinely, such as a time stamp of the application used by the participant and the name of the user action (read, send, etc.) but did not collect data such as passwords or messages.

The experiment was carried out on the participants' Android mobile phone as shown in Figure 2. Ethical approval for this research project was obtained from the university's Research Ethics Committee in order to fulfil University of Plymouth requirements. All the participants were 18 years or older and were asked to read and sign a consent form and information sheet regarding data collection before starting the experiment. In addition, the research and data were conducted and stored within the Centre for Security, Communications and Network Research (CSCAN) at Plymouth University (start date: February 2017; end date: July 2017). Although the study was conducted to collect app log data, no sensitive material was involved. To facilitate a meaningful analysis, at least 100 adult participants (18 years or older) were invited to participate in this metadata capture experiment. Participants took at least one month to complete their participation in the study, during which time they were simply asked to use their device as normal.

For the purpose of the data collection, a code was developed to extract log files from a backup file from the participants' devices after taking a backup after one month on the principal investigator. After one month, each participant's mobile device was connected to the main investigator's computer. Mobile backup was started by utilising Android Debug Bridge (ADB) [27], which is a command line tool that allows communication between the connected Android device and a computer. This necessitated the participating devices having Android OS version 4.1 or above. To access iOS, there is a need to jailbreak the devices to access the log files which unlikely for the users to accept that. On the other hand, android allow to access to mobile detailed and extract data log files without the need to root. Ina addition, to protect the user privacy, ADB was used instead of asking the mobile user to download application.

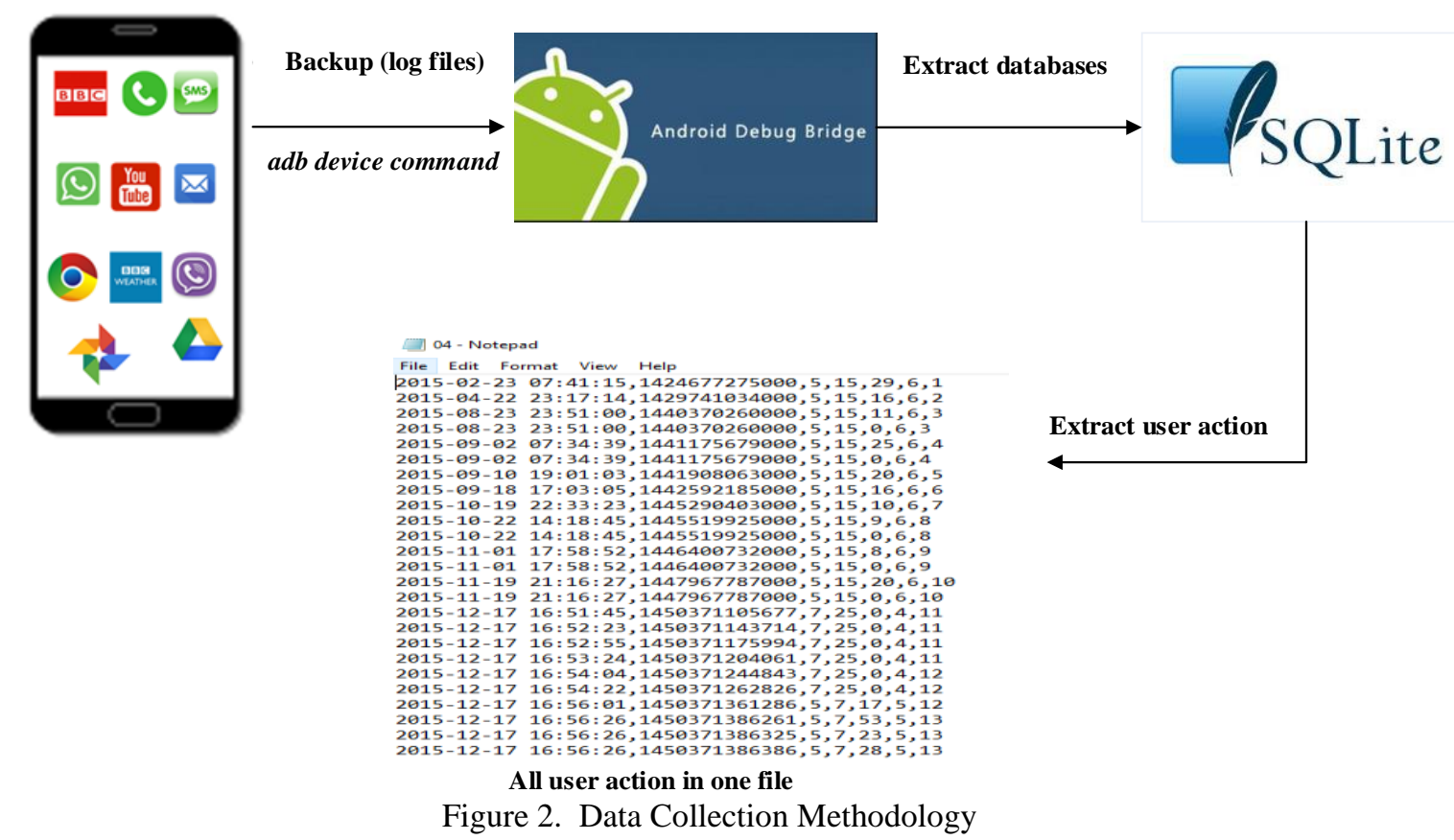


The backup file was extracted and the participant's mobile phone was disconnected. Then, a code was run on SQLite to extract the log files from the extracted backup file. Next, data were generated and the information column was exported to a datasheet file (the time stamp, application name and process name) and stored in a folder called the "UserActionDataSheet". The data were then reviewed by the participant to verify that he/she agreed to share them with the investigator. Finally, the backup file was removed at the end of the experiment period. Although the study is going to collect app log data, there is no sensitive material involved in doing this by writing a code to extract all data automatically once connect the mobile device and protect the user privacy.

During this phase of the data collection, the following applications were selected and collected, as shown in Table 2, and a package name and database name given to each selected application. Some applications, such as Facebook, Online Mobile Banking, and Chrome, were fully encrypted and there was no way of collecting user data without compromising the user's privacy by asking the participant to root his/her device. For this reason, only 12 applications were collected in order to protect the user's privacy.

Table 2. Applications collected from users' mobile phones

\begin{tabular}{|l|l|c|}
\hline App Name & Package name & Database name \\
\hline Phone Call & om.sec.android.provider.logsprovider & logs \\
\hline SMS & com.sec.android.provider.logsprovider & logs \\
\hline Downloading & com.android.providers.downloads & history \\
\hline YouTube & com.google.android.youtube & msgstore \\
\hline WhatsApp & com.whatsapp & SBrowser_Tabs \\
\hline Browser & com.sec.android.app.sbrowser & localappstate \\
\hline Google Play & com.android.vending & EmailProvider \\
\hline Email & com.android.email & viber_data; viber_messages \\
\hline Viber & com.viber.voip & gphotos0_local_media \\
\hline Google Photo & com.google.android.apps.photos & external_Images \\
\hline Camera & com.android.providers.media & external_video \\
\hline Yahoo mail & com.yahoo.mobile.client.android.mail & mailsdk_messages \\
\cline { 2 - 3 } & &
\end{tabular}

\section{Mobile Dataset Analysis and Discussion}

At the end of the data collection, the 76 users had completed the process and the analysis phase was ready to begin. Each user's data were stored in an individual text file, each record containing the following fields: the date (in two forms: human time and a timestamp e.g., 2016-06-28 20:22:30, 1467141750071), application name, action type, and extra information, such as message/email length and call duration. In this study, a total of
$3,015,339$ actions with total usage daily 22457 was accumulated where the long total usage day was 1230 days and 35 was the short total usage day. Table 2 demonstrate the outcomes of such real time behavioural data collection by summering a 47 user action were gathered from 12 applications of 76 participants as shown in Table 3 . In this context, the long total usage day was 1230 days, and 35 was the short total usage day. This, in turn, means that the large dataset sample size might lead to a high degree of accuracy, which would have a positive impact on the conclusions drawn from the proposed approach.

Table 3. 47 User actions collected

\begin{tabular}{|l|l|l|l|l|l|}
\hline No. & Application & Action & No. & Application & Action \\
\hline 1 & Phone Call & Make a Call & 25 & Google Play & Download app \\
\hline 2 & Phone Call & Receive a Call & 26 & Google Play & Update app \\
\hline 3 & SMS & Read a SMS message & 27 & Email & Send an email \\
\hline 4 & SMS & Send a SMS message & 28 & Email & Read an email \\
\hline 5 & Downloading & Download a file & 29 & Viber & Make a free voice Call \\
\hline
\end{tabular}




\begin{tabular}{|l|l|l|l|l|l|}
\hline No. & Application & Action & No. & Application & Action \\
\hline 6 & YouTube & Search on YouTube & 30 & Viber & Make a video Call \\
\hline 7 & WhatsApp & Receive a text message & 31 & Viber & Receive a voice Call \\
\hline 8 & WhatsApp & Receive an image message & 32 & Viber & Receive a video Call \\
\hline 9 & WhatsApp & Receive an audio message & 33 & Viber & Receive a text message \\
\hline 10 & WhatsApp & Receive a video message & 34 & Viber & Receive an image message \\
\hline 11 & WhatsApp & Receive a contact card & 35 & Viber & Receive a sound message \\
\hline 12 & WhatsApp & Receive a location & 36 & Viber & Receive a location \\
\hline 13 & WhatsApp & Receive a free call & 37 & Viber & Send a text message \\
\hline 14 & WhatsApp & Receive a PDF file & 38 & Viber & Send an image message \\
\hline 15 & WhatsApp & Send a text message & 39 & Viber & Send a sound message \\
\hline 16 & WhatsApp & Send an image message & 40 & Viber & Send a location \\
\hline 17 & WhatsApp & Send an audio message & 41 & Viber & Delete a message \\
\hline 18 & WhatsApp & Send a video message & 42 & Google Photo & Upload an image \\
\hline 19 & WhatsApp & Send a contact card & 43 & Google Photo & Upload a video \\
\hline 20 & WhatsApp & Send a location & 44 & Camera & Take a photo \\
\hline 21 & WhatsApp & Make a free call & 45 & Camera & record a Video \\
\hline 22 & WhatsApp & Send a PDF file & 46 & Camera & Save a photo \\
\hline 23 & Browser & Search & 47 & Camera & Save a Video \\
\hline 24 & Browser & Watch a video & & \\
\hline
\end{tabular}

In this section, the overview of the acquired dataset was presented. The histogram in Figure 3 highlights the differences that might be considered significant compared with the total population. For instance, the user profile for participant 71 can be differentiated from the others due to the mobile

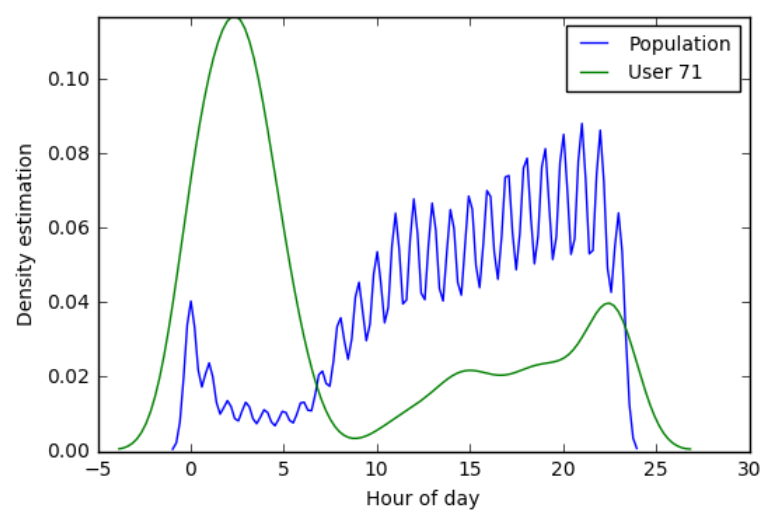

phone mainly being used from 00:00 AM until 6:00 $\mathrm{AM}$, whereas the majority of participants used their mobile phones from 8:00 AM to 10:00 PM. On the other hand, four participants $(42,47,53$, and 68) show identical usage compared with the population.

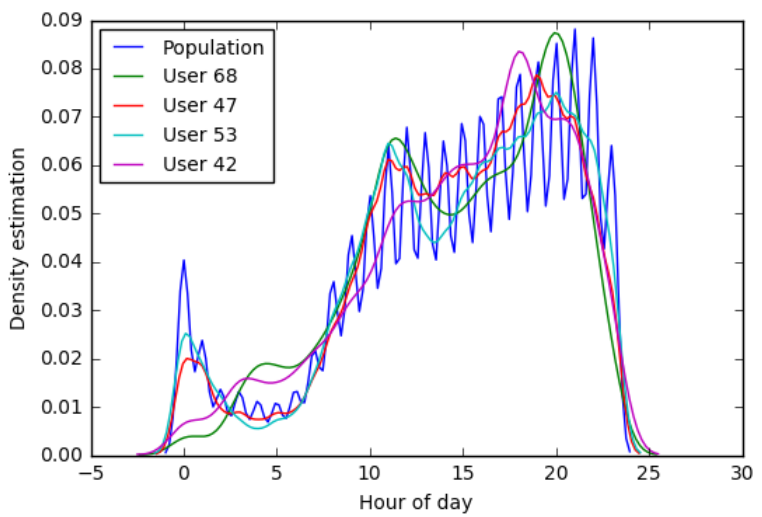

Figure 3: Histogram for population compared with partipents

Figures 4 show that a different sample of behavioral profiling for different users. It is clear from figure 4 and 5 that participant 35 behave like participant 48 regarding the time of mobile applications usage whereas the difference it is very clear between participant 71 and 43 in figures 6 and 7 respectively. Also, participant 11 conduct the same behavioural profiling in most time of usage compared with participant 74 as shown in figures 8 and 9 respectively. In addition, the overall final captured dataset statistics from the 76 participants are summarized and presented in Table 4. This amount of information was felt to be sufficiently rich to allow meaningful analysis; that is, 22,457 days of mobile usage. 


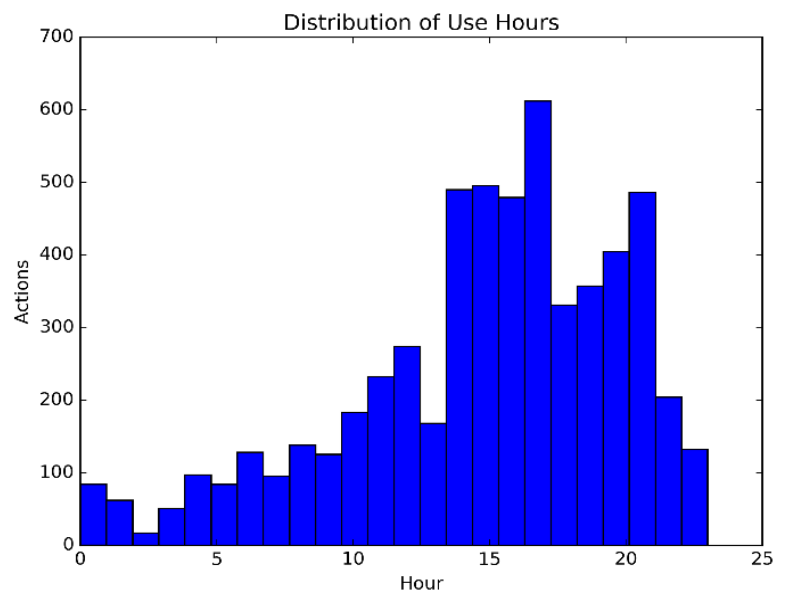

Figure. 4 user 35 distribution

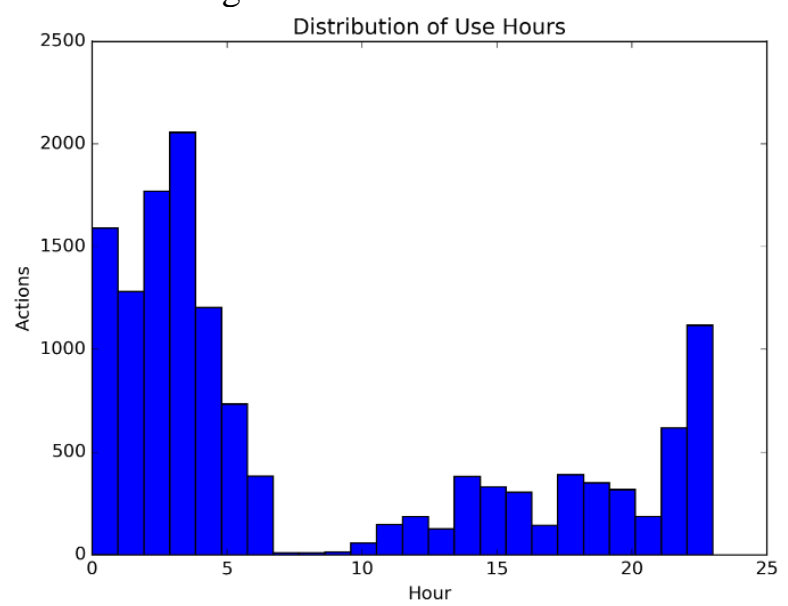

Figure.6 user 71 distribution

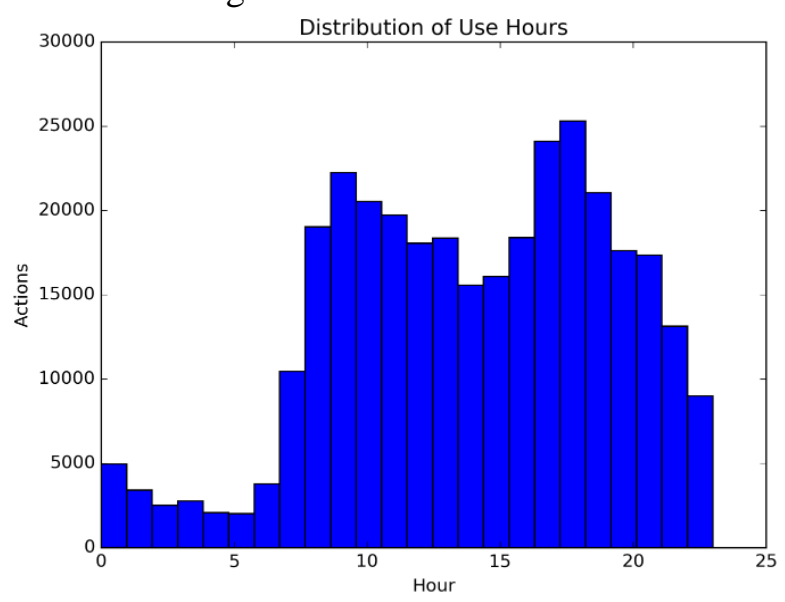

Figure. 8 user 11 distribution

Table 5 shows how many sample points there were for each application. It is clear from the table that WhatsApp was the most frequently accessed application, whereas the other applications taken together were accessed a total of 252,770 times. In this context, the five most commonly used applications among the participants were WhatsApp, Google Play, SMS, Email, and Browser. Although the Viber app was ranked

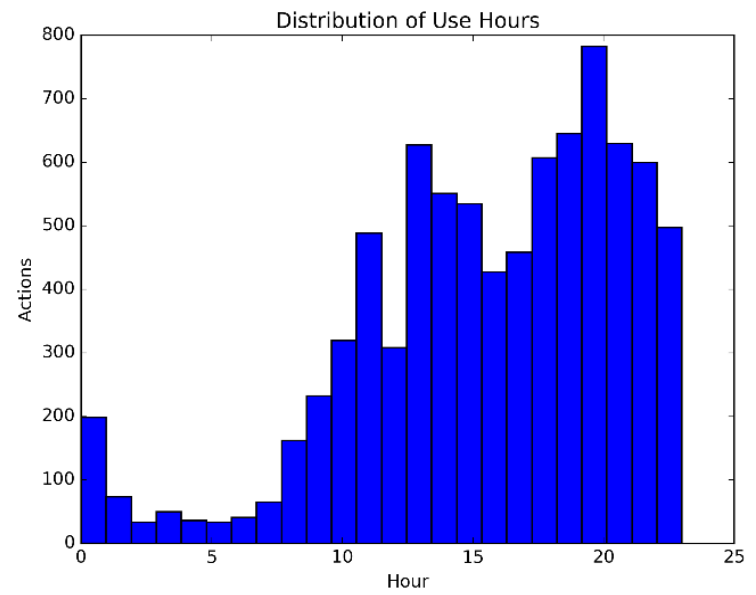

Figure 5. user 48 distribution

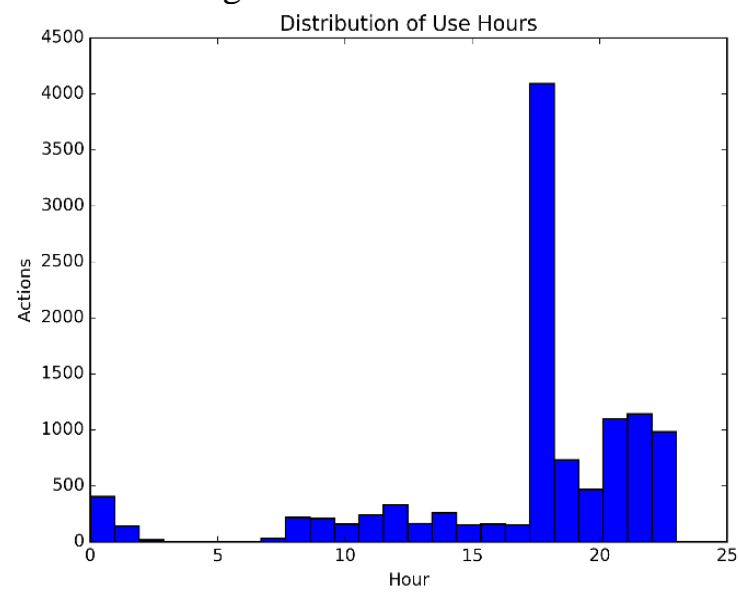

Figure.7 user 43 distribution

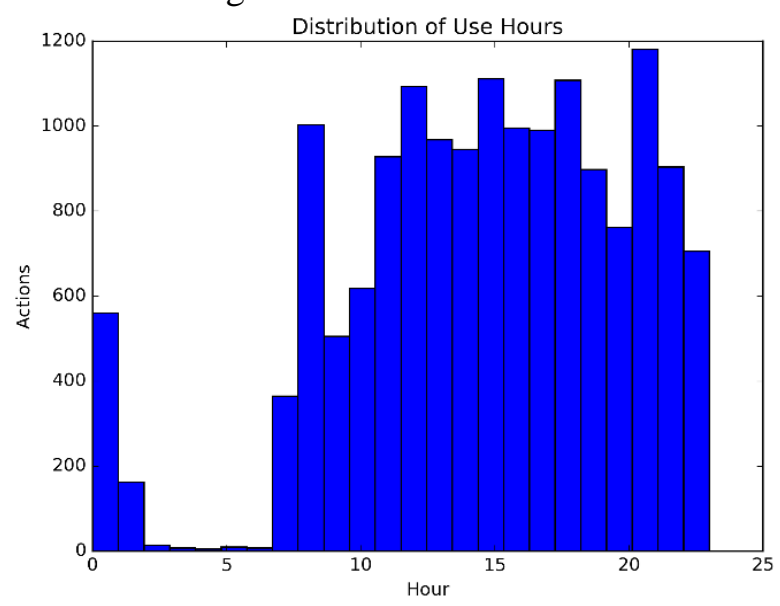

Figure. 9 user 74 distribution

second to WhatsApp in the application samples, with 118,426, as shown in Table 5, it was not commonly accessed among the participants as a whole. 
Table 4: Overall final captured dataset statistics

\begin{tabular}{|l|l|}
\hline Total Number of Users & 76 \\
\hline Total Number of Days & 22,457 \\
\hline Average Number of Days per User & 136 \\
\hline Total Number of Voice Calls & 101,882 \\
\hline Length of Voice Calls & 36,566 hours \\
\hline Total Number of SMS Messages & $2,598,164$ messages \\
\hline Length of SMS Messages & $124,117,633$ characters \\
\hline Total Number of Email Messages & 14,289 email messages \\
\hline Length of Email Messages & $2,813,960$ characters \\
\hline Total Number of Actions Accessed & $3,006,092$ \\
\hline
\end{tabular}

Table 5 demonstrates the total number of actions for each user for the selected applications in this dataset. It is clear from the table that the top three

Table 5: Total number of applications accessed ranked user actions were for WhatsApp. This in turn means that WhatsApp gained the highest amount of usage among all the participants.

\begin{tabular}{|c|c|}
\hline Application Name & Total Number of Times Accessed \\
\hline WhatsApp & $2,753,322$ \\
\hline Viber & 118,426 \\
\hline Google Photo & 49,578 \\
\hline Camera & 25,261 \\
\hline Email & 14,289 \\
\hline Phone Call & 13,808 \\
\hline Browser & 10,785 \\
\hline SMS & 8,459 \\
\hline Downloading & 8,341 \\
\hline Google Play & 3,251 \\
\hline YouTube & 572 \\
\hline
\end{tabular}

On the other hand, Table 6 demonstrates that some user action statistics regarding the 47 user actions collected. It is clear from this table that the majority of those actions were came from WhatsApp application.

Table 6: User action statistics

\begin{tabular}{|l|l|l|l|}
\hline Action Name & Total & Action Name & Total \\
\hline Receive a text message_WhatsApp & $1,662,768$ & Send a text message_WhatsApp & 824,207 \\
\hline Receive image message_WhatsApp & 117,413 & Make a free video Call_Viber & 58,784 \\
\hline Send free sound message_Viber & 49,578 & Receive a video message_WhatsApp & 45,191 \\
\hline Receive image message_Viber & 43,946 & Send an image message_WhatsApp & 40,939 \\
\hline Receive audio message_WhatsApp & 25,753 & Send a location_Viber & 23,308 \\
\hline Take a photo_Camera & 23,308 & Send an email_Email & 13,965 \\
\hline Receive a free call (voice/video) & 12,451 & Search_Browser & 10,643 \\
\hline Make free call(voice/video)WhatsApp & 9,751 & Download a file_Downloading & 8,341 \\
\hline Make a call_Phone Call & 7,606 & Receive a sound message_Viber & 6,28 \\
\hline Receive a call_Phone Call & 6,202 & Send a video message_WhatsApp & 6,029 \\
\hline Send an audio message_WhatsApp & 5,970 & Receive a free voice call_Viber & 5,144 \\
\hline Read a SMS message_SMS & 5,101 & Send an SMS message_SMS & 3,358 \\
\hline Download app_Google Play & 3,251 & Receive a location_Viber & 2,779 \\
\hline Upload image_Google Photo & 1,130 & Save a photo_Camera & 1,130 \\
\hline Receive a free video call_Viber & 1,066 & Receive a PDF file_WhatsApp & 1,016 \\
\hline Receive a contact card_WhatsApp & 842 & Delete a message_Viber & 822 \\
\hline Record a video_Camera & 822 & Search on YouTube_YYouTube & 572 \\
\hline
\end{tabular}




\begin{tabular}{|l|l|l|l|}
\hline Action Name & Total & Action Name & Total \\
\hline Receive a location_WhatsApp & 517 & Read an email_Email & 325 \\
\hline Update app_Google Play & 324 & Send a contact card_WhatsApp & 192 \\
\hline Send a PDF file_WhatsApp & 162 & Watch a video_Browser & 142 \\
\hline Make a free voice call_Viber & 139 & Send a location_WhatsApp & 121 \\
\hline Send free image message_Viber & 10 & Send a free text message_Viber & 4 \\
\hline Receive a text message_Viber & 1 & Upload video_Google Photo & 1 \\
\cline { 1 - 2 } Save a video_Camera & 1 & & \\
\hline
\end{tabular}

Table 7 presents an overview of all the users' data and data collection statistics, which are arranged based on the actions per hour for each user. Each user's data were stored in an individual text file and each record contains the following fields: a date in two formats: human time and a time stamp (e.g., 2016-06-28 20:22:30,
1467141750071), application name, action type, and extra information, such as message/email length and call duration. As illustrated in Table 7, a large amount of user actions took place over a small number of days, as was the case with User ID (UID) 42, which suggests that this individual might be considered a very active user.

Table 7. Data collection statistics

\begin{tabular}{|l|l|l|l|l|l|l|l|l|l|}
\hline U & TA & TUD & APD & APH & U & TA & TUD & APD & APH \\
\hline 11 & 327,476 & 662 & 494 & 20 & 41 & 12,325 & 132 & 93 & 3 \\
\hline 04 & 391,479 & 737 & 531 & 22 & 51 & 17,715 & 187 & 94 & 3 \\
\hline 42 & 16,707 & 40 & 417 & 17 & 07 & 14,741 & 174 & 84 & 3 \\
\hline 47 & 265,603 & 617 & 430 & 17 & 69 & 3,832 & 45 & 85 & 3 \\
\hline 53 & 264,999 & 582 & 455 & 18 & 06 & 19,366 & 236 & 82 & 3 \\
\hline 03 & 96,058 & 284 & 338 & 14 & 30 & 33,956 & 442 & 76 & 3 \\
\hline 67 & 120,757 & 403 & 299 & 12 & 49 & 19,757 & 259 & 76 & 3 \\
\hline 68 & 18,340 & 64 & 286 & 12 & 10 & 36,284 & 487 & 74 & 3 \\
\hline 26 & 3,330 & 14 & 237 & 10 & 32 & 17,605 & 236 & 74 & 3 \\
\hline 28 & 194,615 & 807 & 241 & 10 & 24 & 50,297 & 689 & 73 & 3 \\
\hline 43 & 11,136 & 40 & 278 & 11 & 62 & 10,296 & 143 & 72 & 3 \\
\hline 52 & 28,155 & 102 & 276 & 11 & 73 & 5,600 & 80 & 70 & 3 \\
\hline 71 & 13,702 & 51 & 268 & 11 & 70 & 20,860 & 298 & 70 & 3 \\
\hline 56 & 10,608 & 49 & 216 & 9 & 19 & 19,798 & 346 & 57 & 2 \\
\hline 57 & 56,348 & 261 & 215 & 9 & 37 & 21,587 & 384 & 56 & 2 \\
\hline 09 & 12,256 & 62 & 197 & 8 & 22 & 18,457 & 337 & 54 & 2 \\
\hline 45 & 12,370 & 68 & 181 & 7 & 44 & 34,202 & 637 & 53 & 2 \\
\hline 74 & 15,842 & 81 & 195 & 8 & 55 & 22,933 & 424 & 54 & 2 \\
\hline 34 & 14,645 & 85 & 172 & 7 & 33 & 29,018 & 549 & 52 & 2 \\
\hline 48 & 5,728 & 35 & 163 & 6 & 29 & 14,254 & 285 & 50 & 2 \\
\hline 63 & 15,725 & 94 & 167 & 7 & 05 & 61,205 & 1230 & 49 & 2 \\
\hline 76 & 28,486 & 165 & 172 & 7 & 21 & 21,880 & 443 & 49 & 2 \\
\hline 15 & 16,964 & 107 & 158 & 6 & 50 & 21,269 & 431 & 49 & 2 \\
\hline 36 & 46,917 & 323 & 145 & 6 & 66 & 47,319 & 984 & 48 & 2 \\
\hline 39 & 24,004 & 160 & 150 & 6 & 46 & 27,576 & 592 & 46 & 1 \\
\hline 60 & 22,207 & 149 & 149 & 6 & 38 & 14,807 & 333 & 44 & 1 \\
\hline 64 & 10,822 & 70 & 154 & 6 & 72 & 15,068 & 346 & 43 & 1 \\
\hline 12 & 8,759 & 62 & 141 & 5 & 08 & 14,986 & 343 & 43 & 1 \\
\hline 75 & 5,905 & 46 & 128 & 5 & 61 & 14,496 & 361 & 40 & 1 \\
\hline 02 & 14,412 & 114 & 126 & 5 & 23 & 6,970 & 175 & 39 & 1 \\
\hline 13 & 39,956 & 319 & 125 & 5 & 40 & 10,314 & 261 & 39 & 1 \\
\hline 20 & 21,439 & 168 & 127 & 5 & 59 & 4,967 & 138 & 35 & 1 \\
\hline 14 & 24,140 & 211 & 114 & 4 & 25 & 4,226 & 131 & 32 & 1 \\
\hline 54 & 30,197 & 262 & 115 & 4 & 16 & 20,378 & 644 & 31 & 1 \\
\hline 31 & 20,986 & 195 & 107 & 4 & 35 & 8,394 & 271 & 30 & 1 \\
\hline 65 & 7,081 & 69 & 102 & 4 & 18 & 8,390 & 280 & 29 & 1 \\
\hline 27 & 8,992 & 91 & 98 & 4 & 58 & 10,079 & 349 & 28 & 1 \\
\hline & & & & & & & & & \\
\hline
\end{tabular}




\begin{tabular}{|l|l|l|l|l|l|l|l|l|l|}
\hline U & TA & TUD & APD & APH & U & TA & TUD & APD & APH \\
\hline 01 & 29,463 & 308 & 95 & 3 & 17 & 23,523 & 818 & 28 & 1 \\
\hline U & User ID & \\
\hline TA & Total Actions \\
\hline TUD & Total Usage Days \\
\hline APD & Actions per day \\
\hline APH & Actions per hour \\
\hline
\end{tabular}

\section{Conclusions}

This study presented a new user-apps interactions dataset for behavioural profiling using smartphones in terms of mobile application security. In this research work, a study involving data collected from 76 users over a 1-month period was conducted, generating over 3 million actions based on users' interactions with their smartphone. Furthermore, 12 applications were collected with respect the user's privacy. This dataset might help researches in different aspects. For instance, studying the user behaviour interaction regarding application time usage, user authentication based on behavioural profiling biometric, prediction the next app usage to fulfil both security and usability requirements, and prediction the next action selected as well. For future work, solutions could be suggested and tested to improve the usability and security requirements of the mobile security. More specifically, data on user behaviour is gathered in the background without requiring any dedicated activity by the user, by regularly and periodically checking user behaviour in order to continuously monitor the protection of the mobile device.

\section{References}

[1] Statista (2018) Number of smartphone users worldwide 2014-2020. Available at: https://www.statista.com/statistics/330695/number-ofsmartphone-users-worldwide/ (Accessed: 21 November 2018).

[2] Statista (2016a) Forecast of smartphone user numbers in the United Kingdom) 2015-2022. Available at: https://www.statista.com/statistics/553464/predictednumber-of-smartphone-users-in-the-united-kingdom-uk/ (Accessed: 4 September 2018).

[3] Statista (2016b) World wide mobile app revenues in 2015, 2016 and 2020 (in billion U.S. dollars). Available at:https://www.statista.com/statistics/269025/worldwidemobile-app-revenue-forecast/ (Accessed: 21 November 2018).

[4] Comscore (2015) Mobile Metrix Reveals the UKs Top Smartphone and Tablet Destinations. Available at: https://www.comscore.com/Insights/Data-Mine/Mobile-
Metrix-Reveals-the-UKs-Top-Smartphone-and-TabletDestinations (Accessed: 29 March 2015).

[5] Clarke, N. (2011) Transparent User Authentication: Biometrics, RFID and Behavioural Profiling. London: Springer Science \& Business Media. doi: 10.1007/978-085729-805-8.

[6] Alotaibi, S., Furnell, S. and Clarke, N. (2015) 'Transparent authentication systems for mobile device security: A review', in 2015 10th International Conference for Internet Technology and Secured Transactions (ICITST). IEEE, pp. 406-413. doi: 10.1109/ICITST.2015.7412131.

[7] Crawford, H., Renaud, K. and Storer, T. (2013) 'A framework for continuous, transparent mobile device authentication', Computers \& Security, 39, pp. 127-136. doi: 10.1016/j.cose.2013.05.005.

[8] Traoré, I. and Ahmed, A. A. E. (2012) 'Introduction to Continuous Authentication', in Traore, I. and Ahmed, A. A. E. (eds) Continuous Authentication Using Biometrics. IGI Global, pp. 1-22. doi: 10.4018/978-161350-129-0.ch001.

[9] Clarke, N., Karatzouni, S. and Furnell, S. (2009) 'Flexible and Transparent User Authentication for Mobile Devices', in, pp. 1-12. doi: 10.1007/978-3-64201244-0_1.

[10] Zhang, J. et al. (2018) 'T2FA: Transparent TwoFactor Authentication', IEEE Access. IEEE, 6, pp. 32677-32686. doi: 10.1109/ACCESS.2018.2844548.

[11] Hatin, J. et al. (2017) 'Privacy Preserving Transparent Mobile Authentication', in Proceedings of the 3rd International Conference on Information Systems Security and Privacy. SCITEPRESS - Science and Technology Publications, pp. 354-361. doi: 10.5220/0006186803540361.

[12] Meng, W. et al. (2015) 'Surveying the Development of Biometric User Authentication on Mobile Phones', IEEE Communications Surveys \& Tutorials. IEEE, 17(3), pp. 1268-1293. doi: 10.1109/COMST.2014.2386915.

[13] Mahfouz, A., Mahmoud, T. M. and Eldin, A. S. (2017) 'A survey on behavioral biometric authentication on smartphones', Journal of Information Security and Applications, 37, pp. 28-37. doi: 10.1016/j.jisa.2017.10.002. 
[14] Li, F. et al. (2011) 'Misuse Detection for Mobile Devices Using Behaviour Profiling', International Journal of Cyber Warfare and Terrorism, 1(1), pp. 4153. doi: 10.4018/ijcwt.2011010105.

[15] Eagle, N. and Pentland, A. (2006) 'Reality mining: sensing complex social systems', Personal and Ubiquitous Computing, 10(4), pp. 255-268. doi: 10.1007/s00779-005-0046-3.

[16] Li, F. et al. (2014) 'Active authentication for mobile devices utilising behaviour profiling', International Journal of Information Security, 13(3), pp. 229-244. doi: 10.1007/s10207-013-0209-6.

[17] Saevanee, H., Clarke, N. L. and Furnell, S. M. (2012) 'Multi-modal Behavioural Biometric Authentication for Mobile Devices', in 27th IFIP International Information Security and Privacy Conference - SEC2012, Heraklion, Crete, Greece, 4-6 June, pp465-474, 2012, pp. 465-474. doi: 10.1007/9783-642-30436-1_38.

[18] Saevanee, H. et al. (2014) 'Text-Based Active Authentication for Mobile Devices', in, pp. 99-112. doi: 10.1007/978-3-642-55415-5_9.

[19] Fridman, L. et al. (2017) 'Active Authentication on Mobile Devices via Stylometry, Application Usage, Web Browsing, and GPS Location', IEEE Systems Journal, 11(2), pp. 513-521. doi: 10.1109/JSYST.2015.2472579.

[20] Neal, T. J. and Woodard, D. L. (2017) 'Using associative classification to authenticate mobile device users', in 2017 IEEE International Joint Conference on Biometrics (IJCB). IEEE, pp. 71-79. doi: 10.1109/BTAS.2017.8272684.

[21] Shi, W. et al. (2011) 'SenGuard: Passive user identification on smartphones using multiple sensors', in 2011 IEEE 7th International Conference on Wireless and Mobile Computing, Networking and Communications (WiMob). IEEE, pp. 141-148. doi: 10.1109/WiMOB.2011.6085412.

[22] Khan, H. and Hengartner, U. (2014) 'Towards application-centric implicit authentication on smartphones', in Proceedings of the 15th Workshop on Mobile Computing Systems and Applications HotMobile '14. New York, New York, USA: ACM Press, pp. 1-6. doi: 10.1145/2565585.2565590.

[23] Hayashi, E. et al. (2012) 'Goldilocks and the Two Mobile Devices: Going Beyond All-Or-Nothing Access to a Device's Applications', in Proceedings of the Eighth Symposium on Usable Privacy and Security. New York, New York, USA: ACM Press. doi: $10.1145 / 2335356.2335359$.

[24] Gupta, S., Buriro, A. and Crispo, B. (2018) 'Demystifying Authentication Concepts in Smartphones: Ways and Types to Secure Access', Mobile Information Systems, 2018, pp. 1-16. doi: 10.1155/2018/2649598.
[25] Kayacik, H. G. et al. (2014) 'Data Driven Authentication: On the Effectiveness of User Behaviour Modelling with Mobile Device Sensors', in Proceedings of the Mobile Security Technologies Workshop. Available at: http://arxiv.org/abs/1410.7743 (Accessed: 9 April 2019).

[26] Acien, A., Morales, A., Vera-Rodriguez, R. and Fierrez, J. (2019) 'MultiLock: Mobile Active Authentication based on Multiple Biometric and Behavioral Patterns'. Available at: http://arxiv.org/abs/1901.10312.

[27] Android (2019) Android Debug Bridge (adb). Available at:

https://developer.android.com/studio/command-line/adb (Accessed: 2 January 2019)

[28] Tan, C., Liu, Q., Chen, E. and Xiong, H., (2012). Prediction for mobile application usage patterns. In Nokia MDC workshop (Vol. 12).

[29] Huang, K., Zhang, C., Ma, X., \& Chen, G. (2012, September). Predicting mobile application usage using contextual information. In Proceedings of the 2012 ACM Conference on Ubiquitous Computing (pp. 1059-1065). ACM.

[30] Yan, T., Chu, D., Ganesan, D., Kansal, A., \& Liu, J. (2012, June). Fast app launching for mobile devices using predictive user context. In Proceedings of the 10th international conference on Mobile systems, applications, and services (pp. 113-126). ACM.

[31] Zou, X., Zhang, W., Li, S., \& Pan, G. (2013, September). Prophet: What app you wish to use next. In Proceedings of the 2013 ACM conference on Pervasive and ubiquitous computing adjunct publication (pp. 167170). ACM.

[32] Liao, Z. X., Lei, P. R., Shen, T. J., Li, S. C., \& Peng, W. C. (2012, December). Mining temporal profiles of mobile applications for usage prediction. In 2012 IEEE 12th International Conference on Data Mining Workshops (pp. 890-893). IEEE.

[33] Pan, W., Aharony, N., \& Pentland, A. (2011, August). Composite social network for predicting mobile apps installation. In Twenty-Fifth AAAI Conference on Artificial Intelligence.

[34] Krishnaswamy, S., Gama, J., \& Gaber, M. M. (2012, July). Mobile data stream mining: from algorithms to applications. In 2012 IEEE 13th International Conference on Mobile Data Management (pp. 360-363). IEEE. 